\title{
In Memoriam: Professor Merle Brown . Daniel Bachhuber
}

You said, "The poet

Always listens to himself

As part of the expressive act

That is his poem."

I know what that means.

The listening tells us

How much of our own lives

We must love enough to save. 